\title{
Formalization of Initial Requirements for the Design of Wireless Sensor Networks
}

\author{
Kislyakov, M.A. \\ Vladimir State University named after \\ Alexander and Nikolay Stoletovs, VSU \\ Vladimir, Russian Federation \\ kislyakov.maxim@gmail.com
}

\author{
Mosin, S.G. \\ Vladimir State University named after \\ Alexander and Nikolay Stoletovs, VSU \\ Vladimir, Russian Federation \\ smosin@ieee.org
}

\begin{abstract}
The paper discusses methods for the formal representation of the initial requirements for wireless sensor networks at the design stage. The main objective of the formalization is generating the basic mathematical tool and optimization criteria for individual stages of the design process. The automation of the design procedures with the use of computer technology is planned in terms of the proposed basis.
\end{abstract}

Keywords - wireless sensor network; formalization; automation; optimization criterion

\section{INTRODUCTION}

Wireless sensor networks (WSN) are among the most relevant technologies at present. The applications of this technology are systematized, classified and include such basic directions as military defense, agriculture, industry, medicine and household sector [2,3]. Moreover, the significance of the systems based on sensor networks is high in developed as well as in developing countries [1].

Currently, there are several manufacturers who offer their solutions on the market. Such solutions have a number of technical parameters which determine the range of use. It should be noted that the existing hardware and software complexes of sensor networks are created with the use of computer technology, but only individual stages of the design process are subject to automation. Consequently, the complex automation of the design procedure set is a relevant issue.

The task of automating the WSN design process involves the generation of the flow to be performed by the system under the supervision of a developer. In other words, the automation system is a software implementation of the design flow, where the developer is only a user.

The design of complex objects is based on the application of ideas and principles presented in a number of theories and approaches. The most common one is the system approach which determines the number of automation system components [4]. Firstly, the system must have a hierarchical structure. The hierarchical representation allows identifying a number of subsystems that can be designed independently. Secondly, the system must include analysis and simulation procedures. The purpose of these procedures is to identify a set of object parameters which will allow deciding on the next stage. Thirdly, the system must contain synthesis and optimization procedures which solve the problems of generating and modifying the object in order to achieve the parameters meeting the optimization criteria.

The automation design system allows for the development of the target object, but this is impossible without the formation of fixed criteria. Such criteria may be formed only on the basis of a number of a developer's initial requirements to the designed object. However, such requirements have a useroriented format that is not suitable for the system. Therefore, the formalization of initial parameters is necessary.

The paper presents methods for the formalization of the initial requirements to a designed WSN object. The structure of the paper includes four sections. Section II contains a brief description of the design flow of WSN, according to which the optimization criteria based on the initial requirements for an object are proposed. Five key parameters that must be entered into the system by the user are identified in Section III. The proposed number of parameters is considered as sufficient to form a complete network object. Section IV concludes the paper.

\section{DESIGN FLOW OF WSN}

A detailed description of the design flow of WSN is not the purpose of this paper, but the general idea of the design process is necessary.

The automation design system is primarily determined by its flow. The flow determines the order of the design procedures and all the transitions between them. Consider the sequence of procedures applicable to WSN design.

The design flow of WSN comprises the following steps:

- $\quad$ Entering the initial requirements for WSN;

- Determination of a component basis;

- The synthesis of the basic structure;

- $\quad$ The use of the strategy for reliability;

- The use of the strategy for energy efficiency;

- Simulation;

- Generating the final project. 
The stages of providing reliability and energy efficiency are iterative, which determines the presence of synthesis procedures as well as analysis procedures in the form of components. The design flow is also presented as a cycle, which ensures the process of parameter convergence of the designed object to the initial requirements.

\section{FORMALIZATION OF INITIAL REQUIREMENTS}

Entering the initial requirements of the project is the first procedure to be performed. The automation of entering the information is difficult. It is based on the full interaction of the developer and the system. However, the formalization of the input data can be automated with the use of mathematical tool proposed in the paper.

Five main parameters necessary for the design of WSN must be singled out of the set of initial requirements. The parameter list is given below:

- $\quad$ Life cycle of the network;

- $\quad$ Coverage area;

- Degree of information relevance;

- Data transmission rate;

- Level of network reliability.

The initial requirements are the basis for generating the internal parameters of the system. Their formalization is given below.

\section{A. Life cycle of the network}

The life cycle of the network determines the maximum uptime of the designed object. Theoretical calculation of the uptime of the network is presented in (1).

$$
T_{c}=\min \left(T_{y}\right)
$$

where $T_{c}$ is the total uptime of the network, $T_{y}$ is the set of uptime values of the nodes such that $T_{y}=\left\{t_{y 1}, t_{y 2}, \ldots, t_{y n}\right\}$, $n$ is the number of network nodes.

On the other hand, the life cycle of the node corresponds to the number of periods in the schedule (2).

$$
t_{y i}=m_{i} t_{y p}
$$

where $i=\{1 . . n\}$ is the index of the node, $t_{y p}$ is the duration of one period in accordance with the schedule, $m_{i}$ is the number of periods for the $i$-th node.

Five components of $t_{y p}$ must be distinguished:

- $t_{y p 0}-$ sleep period;

- $t_{y p l}-$ waiting period;

- $t_{y p 2}-$ period of reading sensor data;

- $\quad t_{y p 3}-$ period of data reception from another node;

- $\quad t_{y p 4}-$ period of data transmission to another node;
- $t_{y p 5}-$ period of information processing.

Parameter $m_{j}$ matches period $t_{y p j}$. This parameter specifies the number of repetitions of period $t_{y p j}$ for the node. Equation (3) is the calculation of period $t_{y p}$.

$$
t_{y p}=\sum_{j=0}^{5}\left(m_{j} t_{y p j}\right)
$$

Each period $t_{y p j}$ is characterized by its indicator of energy consumption. Thus, parameter $S_{j}$ which determines the discharge rate of the independent power supply unit corresponds to period $t_{y p j}$. In turn, the power supply has a certain capacity of $P$. Let the capacity of power supply for all nodes be equal to $P$. Then the optimization criterion has the form represented by (4).

$$
P \geq \sum_{j=0}^{5}\left(m_{j} t_{y p j} S_{j}\right)
$$

The values of parameters $t_{y p j}$ and $S_{j}$ are known. Parameter $m_{j}$ is calculated based on the simulation of the object. Based on the initial formulation of the problem, the number of schedule periods $m$ corresponds to the number of sleep periods $m_{0}$ of the node. Thus, the parameters of the life cycle of each node in the network can be calculated based on (2) and (4). The total uptime of the network is formed based on (1). Comparing the calculated parameter $T_{c}$ with the initial requirements for the life cycle of the network, the system selects the next stage of the design flow.

\section{B. Coverage area}

The coverage area determines the spatial arrangement of network nodes which perform the role of sensors. Considering it, the network has a static topology, and location of nodes does not change with time. Then the network coverage can be represented as three sets: $X=\left\{x_{1}, \ldots, x_{n}\right\}, Y=\left\{y_{1}, \ldots, y_{n}\right\}$, $Z=\left\{z_{1}, \ldots, z_{n}\right\}$.

Each node contains a transmitter with a certain power level. The power level of the transmitter determines the node action radius $R$ in open space. Let the action radius for all nodes be equal to $R$.

Consider the case where all nodes can act as relays of information. Then the distance between nodes $i$ and $j$ in the three-dimensional space will be calculated by (5).

$$
D_{i j}=\sqrt{\left(x_{i}-x_{j}\right)^{2}+\left(y_{i}-y_{j}\right)^{2}+\left(z_{i}-z_{j}\right)^{2}}
$$

where $i, j=(1 . . n)$ and $i \neq j$.

If the nodes connected to the sensors can not receive the information via radio channel, the relay must be added to the network. In this case, the calculation of the distance between the nodes is performed by (6).

$$
D_{i j}=\sqrt{\left(x_{i}-x_{j}^{\prime}\right)^{2}+\left(y_{i}-y_{j}^{\prime}\right)^{2}+\left(z_{i}-z_{j}^{\prime}\right)^{2}}
$$

where $i=(1 . . n), j=(1 . . l), l$ is the number of relays added to the network, $\left(x_{j}^{\prime}, y_{j}^{\prime}, z_{j}^{\prime}\right)$ are coordinates of the $j$-th relay. It 
should be noted that the relays can be added in the case of equivalent nodes.

To fulfill the initial requirements of the coverage the system should ensure network connectivity. Each node must have at least one route for the transmission of information over the network. This requirement can be expressed by (7) and (8) for equivalent and nonequivalent nodes, respectively.

$$
\begin{gathered}
Q_{i}=\sum_{j=0}^{n} f\left(R \geq D_{i j}\right) \\
Q_{i}=\sum_{j=0}^{l} f\left(R \geq D_{i j}\right)
\end{gathered}
$$

where $f(x)$ is the function of condition calculation. If the condition is satisfied, then $f(x)=1$, otherwise $f(x)=0$. Thus, the network connectivity is provided, if set $Q$ contains no elements equal to zero. If the connectivity condition is not satisfied, an extra relay is added to the network.

\section{Degree of information relevance}

The transmission time from the source to the receiver determines the degree of information relevance. It is considered that for a period of scheduling a network node can receive and transmit no more than one information packet.

Let $M=\left\{m_{l}, \ldots, m_{n}\right\}$ be the set which defines the same route for each node in the network. For each route $m_{i}$ parameter $h_{i}$ is the dimension. The route dimension is numerically equal to the number of relays on the path from the source to the destination node.

Parameter $T_{a}$ is the initial requirement to the degree of information relevance. This parameter is numerically equal to the maximum information transmission time. Then the relevance criterion can be determined by (9).

$$
T_{a} \geq \max (H) \cdot t_{y p}
$$

where $H=\left\{h_{1}, \ldots, h_{n}\right\}$ is a set of route dimensions.

If (9) is fulfilled, the relevance is provided, otherwise generating new routes is necessary.

\section{Data transmission rate}

The user forms the requirements to data transmission rate $S_{p}$ in accordance with the target function of the network. In general terms, the user specifies the maximum transmission rate of each sensor node. The initial parameters are limited only by the bandwidth of the radio channel. However, the routes may overlap using shared relays. Thus, the bandwidth is reduced in proportion to the number of overlapping routes.

The bandwidth of the radio channel is defined as $S_{r}$. The next step is to define set $U=\left\{u_{1}, \ldots, u_{n}\right\}$, where $u_{i}$ - the number of the routes which include the $i$-th network node. Then the initial requirement criterion for the data transfer rate is expressed by (10).

$$
S_{p} \leq S_{r} / \max (U)
$$

If (10) is fulfilled, the transfer rate is provided, otherwise generating new routes is necessary.

It should be noted that the parameters of relevance and data transmission rate are interrelated. Set $H_{m}=\left\{h_{m l}, \ldots, h_{m n}\right\}$ is added. Its elements are calculated according to (11). Then (9) takes the form of (12).

$$
\begin{gathered}
h_{m i}=h_{i} \cdot u_{i} \\
T_{a} \geq \max \left(H_{m}\right) \cdot t_{y p}
\end{gathered}
$$

Consequently, the parameters of relevance and data transmission rate depend on set $M$. Reducing the number of intersections of the routes increases the data transmission rate and reduces the total time of message delivery.

\section{E. Level of network reliability}

The reliability of the network is one of its most important parameters. The failure probability of the network is defined as $P_{c}$. The failure probability of the node is defined as $P_{y}$. Parameter $P_{y}$ is determined by the selected component basis. It is formed on the basis of specifications.

When generating a static network topology the routes can be calculated at the design stage. If each node doesn't use more than one route, then $P_{c}=P_{y}$. If the system provides a number of reserved routes, the value of $P_{c}$ is reduced, but the exact mathematical representation is quite difficult to make. The simplest way to assess the reliability of the process will be running a simulation considering $P_{y}$ and iterative analysis of the network connectivity. The reliability is supposed to be estimated based on statistical data.

\section{CONCLUSION}

The methods for the formal representation of the initial requirements for WSN have been proposed in the paper. Mathematical models of optimization criteria which ensure the convergence of the design process have been examined. The proposed mathematical tool is aimed at automation of the project procedures in accordance with the design flow of WSN.

The development of CAD tools for WSN design based on the proposed mathematical models is the next task of the authors.

\section{REFERENCES}

[1] S. Taruna, Kusum Jain, G.N. Purohit, "Application Domain of Wireless Sensor Network: - A Paradigm in Developed and Developing Counties," IJCSI International Journal of Computer Science Issues, vol. 8, issue 4, no 2, July 2011, pp. 611-617.

[2] I. Khemapech, I. Duncan, A. Miller, "A Survey of Wireless Sensor Networks Technology," in PGNET, Proceedings of the 6th Annual PostGraduate Symposium on the Convergence of Telecommunications, Networking \& Broadcasting, (Liverpool, UK), EPSRC, June 2005.

[3] I.F. Akyildiz, W. Su, Y. Sankarasubramaniam, E. Cayirci, "Wireless Sensor Networks: a Survey," Computer Networks, vol. 38, 2002 , pp. 393-422.

[4] I.P. Norenkov, V.B. Manichev, "Osnovi teorii proektirovaniya SAPR [Basic Theory and Design CAD]," Moscow: High school, 1990, p. 335. (rus). 